\title{
Antitumor and immunomodulating activities of six Phellinus igniarius polysaccharides of different origins
}

\author{
WENWEN GAO ${ }^{1,2}$, WANGDI WANG ${ }^{1}$, WENJIAN SUN ${ }^{1}$, MINGFANG WANG $^{1}$, NA ZHANG $^{1}$ and SHUWEN YU ${ }^{2}$ \\ ${ }^{1}$ Department of Clinical Pharmacy, School of Pharmaceutical Sciences, Shandong University, Jinan, Shandong 250012; \\ ${ }^{2}$ Department of Pharmacy, Jinan Central Hospital Affiliated to Shandong University, Jinan, Shandong 250013, P.R. China
}

Received November 1, 2015; Accepted February 1, 2017

DOI: 10.3892/etm.2017.5191

\begin{abstract}
The aim of the current study was to compare the antitumor efficiency of Phellinus igniarius polysaccharides (PIP) from six different origins and preliminarily investigate its potential mechanisms. PIP was extracted using the microwave extraction method. The corresponding in vivo antitumor efficacy was assessed in Kunming mice bearing H22 tumors and Gansu PIP (GPIP) was identified to have a significantly higher antitumor efficacy compared with the control group $(\mathrm{P}<0.05)$, while no significant difference was observed following treatment with PIP from different origins $(P>0.05)$. The spleen index of the GPIP group significantly increased compared with the saline and CTX groups $(\mathrm{P}<0.01)$. The in vitro MTT assay of GPIP on HepG2 cells indicated that GPIP had no direct cytotoxicity. The serum immune cytokines of interleukin-2, interleukin-12 and interferon- $\gamma$ were assessed using the ELISA method. The concentration of all three serum cytokines significantly increased compared with saline and CTX groups $(\mathrm{P}<0.01)$ indicating that activating the immune system may be a potential antitumor mechanism. These results demonstrated that GPIP has great potential as a natural antitumor agent with immunomodulatory activity.
\end{abstract}

\section{Introduction}

Cancer is a leading cause of mortality worldwide and includes numerous diseases characterized by the uncontrolled proliferation of anaplastic cells, which are able to invade surrounding tissues and metastasize to other organs (1). Many currently available antitumor drugs have been demonstrated to cause intolerable side effects and complications; for instance, doxorubicin may induce severe cardiac toxicity (2). Therefore, it is important to identify natural, less toxic and effective

Correspondence to: Dr Shuwen Yu, Department of Pharmacy, Jinan Central Hospital Affiliated to Shandong University, 105 Jie Fang Road, Jinan, Shandong 250013, P.R. China

E-mail: yushuwen@sdu.edu.cn

Key words: Phellinus igniarius polysaccharides, antitumor, spleen and thymus index, immunomodulation, cytokines products to prevent and treat cancer. For a number of years, humans have benefited from green plants as a source of pharmacological agents and herbal remedies $(3,4)$. For example, mushrooms have attracted attention as a traditional food and medicine for a long time. The polysaccharides of mushrooms were proven to be an effective ingredient for the treatment of diseases such as lung cancer and colorectal carcinoma (5). In addition to mushroom polysaccharides, other polysaccharides isolated from natural materials have also been demonstrated to prevent ailments including gastroenteric dysfunction, diarrhea and cancer $(6,7)$.

Phellinus igniarius (PI), one of the most famous traditional Chinese medicines, is classified into Hymenochaetaceae Basidiomycete, and is widely used in Asia $(8,9)$. PI polysaccharides (PIP), the aqueous extraction of PI, have been demonstrated to have an antitumor bioactivity (10). Previous research into the antitumor mechanisms have focused on the direct inhibition of cancer cells $(11,12)$. However, polysaccharides have attracted more attention due to their immunomodulatory effects $(13,14)$. It has been demonstrated that developing cancer is able to avoid detection and escape the immune response (15). By adjusting or stimulating immune functions, the tumor cells may be recognized and targeted by the immune system (16). As such, immunotherapy has typically been employed in clinical settings to achieve an improved treatment and outcome of cancer (17). Therefore, in the present study, the immunomodulatory effects of PIP were assessed and the potential for PIP to provide antitumor immunotherapy was investigated.

The biological activities of polysaccharides primarily depend on a number of structural features including monosaccharide composition, molecular weight, type of glycosidic bond and branch structures $(18,19)$. For PI, the structure and bioactivity of polysaccharides may be affected by the origin and species. Therefore, in the present study, six different origins or species of PI were collected from China. These PIs were subsequently screened and the one with the best antitumor effect was preliminary studied to assess the antitumor mechanisms. PIP was extracted using a microwave extraction method. The in vivo antitumor efficacy, spleen index and thymus index was assessed in Kunming (KM) mice bearing H22 tumors to identify the PI with the highest antitumor efficacy. The antitumor mechanism was investigated using MTT assay and by testing the concentration of serum immune 
cytokines including interleukin-2 (IL-2), interleukin-12 (IL-12) and interferon- $\gamma(\mathrm{IFN}-\gamma)$.

\section{Materials and methods}

Materials. A total of six types of PI fruiting bodies (including Dongbei mulberry Phellinus igniarius and Dongbei white birch Phellinus igniarius) were obtained from Shandong, Gansu, Hunan, unknown origin and Dongbei province of China. All chemicals and kits were obtained commercially. Cyclophosphamide (CTX) and Trametes versicolor polysaccharopeptide (PSP) were purchased from the Jinan Central Hospital (Jinan, China). HepG2 and murine hepatocellular liver carcinoma (H22) cells were obtained from the Shandong Institute of Immunopharmacology and Immunotherapy (Jinan, China). MTT and RPMI-1640 were purchased from Sigma-Aldrich (Merck KGaA; Darmstadt, Germany). IL-2, IL-12 and IFN- $\gamma$ kits were purchased from Jinan Rebecca Trading Co. (Jinan, China).

Animals. A total of 140 4-week-old female KM mice with a weight of 18-22 $\mathrm{g}$ were supplied by Laboratory Animals Center of Shandong University (Jinan, China). The mice were housed under normal laboratory conditions $\left(24 \pm 2^{\circ} \mathrm{C}, 50 \pm 20 \%\right.$ humidity, 12/12-h light-dark cycle) with free access to standard rodent chow and water. Following acclimatizing to the lighting conditions for 3 days, 132 mice of similar weights were selected for the investigation. The experiment was performed following the guidelines of the Ethical Committee for Animal Experiments of Shandong University. All animal experiments complied with the requirements of the National Act on the Use of Experimental Animals. There were 11 groups in total and 12 mice were used in each group.

Preparation of PIP. A total of six different types of PI fruiting bodies were dried in an oven at $60^{\circ} \mathrm{C}$ for $24 \mathrm{~h}$, ground to powdered-form and kept in an air-tight plastic bag at room temperature until use. To prepare crude polysaccharides, $10 \mathrm{~g}$ of each PI sample was extracted with $500 \mathrm{ml}$ distilled water using microwave extraction for $15 \mathrm{~min}$, three times. The extract was concentrated with RE52-98 rotary evaporation apparatus (Shanghai Ya Rong Biochemical Instrument Factory, Shanghai, China), precipitated with $80 \%$ ethanol and stored at $4^{\circ} \mathrm{C}$ for $24 \mathrm{~h}$. The method of preparation was as previously described (20) and a clear description of the process is demonstrated by the flow diagram presented in Fig. 1. The concentration of total PIP from Dongbei mulberry, Dongbei birch, Gansu mulberry, Hunan mulberry, Shandong mulberry and mulberry from an un-known place were 5.37, 2.21, 3.04, $3.35,2.77$ and $3.58 \%$, respectively (20). The precipitate was subsequently centrifuged at $4^{\circ} \mathrm{C}$ and $860 \mathrm{xg}$ for $15 \mathrm{~min}$, dried in a vacuum and deproteinized using sevage reagent (chloroform; Shanghai Aladdin Bio-Chem Technology Co., Ltd., Shanghai, China).

In vivo tumor growth inhibition study. KM mice implanted with $\mathrm{H} 22$ cells were used to qualify the relative efficacy of six types of PIP through oral administration. All animal experiments were performed in full compliance with guidelines approved by the Animal Care Committee of Shandong University.

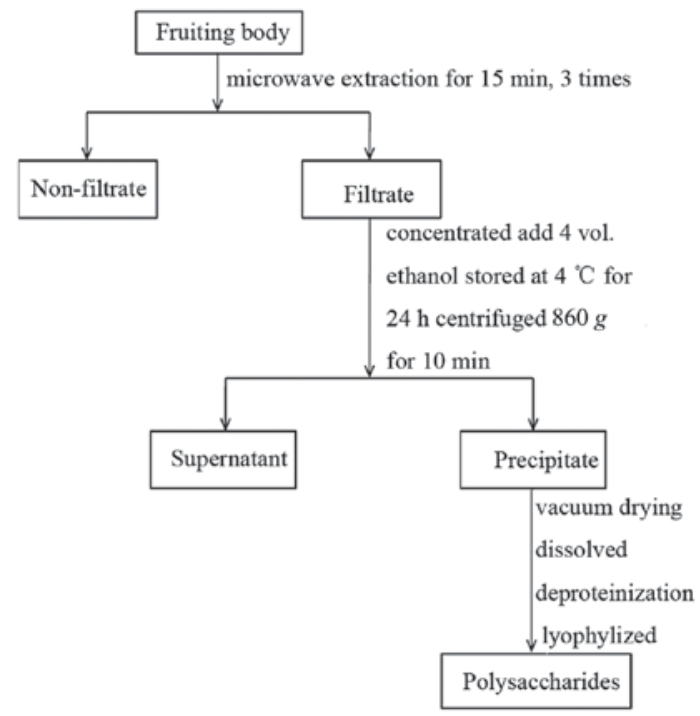

Figure 1. Flow chart of extraction for Phellinus igniarius polysaccharides.

All 132 mice, including the control group, were subcutaneously injected in the right axillary space with $1 \times 10^{7} \mathrm{H} 22$ cells to establish the hepatoma model mice at day 0 . Treatments were started $24 \mathrm{~h}$ following tumor cell injection.

On Day 0 , the mice were randomly divided into 11 groups with 12 mice in each which received the following treatments: Group i) saline; group ii) CTX (intraperitoneal injection; CTX concentration of $25 \mathrm{mg} / \mathrm{kg}$; diluted in saline); group iii): PSP (intragastrically; $200 \mathrm{mg} / \mathrm{kg}$; diluted in distilled water); groups iv-ix): Dongbei mulberry Phellinus igniarius polysaccharide (DMPIP), Hunan mulberry Phellinus igniarius polysaccharide (HPIP), Gansu mulberry Phellinus igniarius polysaccharide (GPIP), Dongbei white birch Phellinus igniarius polysaccharide (DWPIP), Shandong mulberry Phellinus igniarius polysaccharide (SPIP) and unknown origin Phellinus igniarius polysaccharide (UPIP), respectively (intragastrically; $200 \mathrm{mg} / \mathrm{kg}$; diluted in distilled water); group x): GPIP plus CTX at the same time; group xi): PSP plus CTX. The treatments were administered once daily for two weeks. All mice were tagged and tumor size was measured daily with calipers during the period of study. The tumor volume was calculated according to the following formula: $\left(\mathrm{W}^{2} \mathrm{x} \mathrm{L}\right) / 2$, where $\mathrm{W}$ is the tumor measurement at the widest point and $\mathrm{L}$ is the tumor dimension at the longest point. Each mouse was weighed at the time of treatment, so that dosages were adjusted to achieve the correct $\mathrm{mg} / \mathrm{kg}$ amounts. The body weights of mice were monitored as an index of systemic toxicity. At the end of the experiment, the animals were sacrificed by cervical dislocation, and the tumor, spleen and thymus were harvested by dissection and weighed. The tumor inhibition ratio was calculated according to the following equation: Inhibition rate (\%) $=$ (mean tumor weight of saline group-mean tumor weight of treated group)/mean tumor weight of saline group x100. The thymus and spleen index were calculated using the following formula: Thymus or spleen index $=$ the weight of the thymus or spleen/total weight of mouse.

Cell culture. HepG2 cells were grown in RPMI-1640 medium containing 10\% fetal bovine serum (Sigma-Aldrich; Merck 
Table I. Effect of PIP on the number of living mice, tumor weight and inhibitory rate of $\mathrm{H} 22$ cells.

\begin{tabular}{lcccc}
\hline & \multicolumn{2}{c}{$\begin{array}{c}\text { Total no. } \\
\text { of mice }\end{array}$} & & \\
\cline { 2 - 3 } Groups & Before & After & Tumor weight, g & Inhibition, \% \\
\hline Saline & 12 & 12 & $0.798 \pm 0.214$ & \\
CTX & 12 & 10 & $0.234 \pm 0.050^{\mathrm{b}}$ & 70.68 \\
PSP & 12 & 12 & $0.493 \pm 0.230^{\mathrm{a}}$ & 38.22 \\
DMPIP & 12 & 11 & $0.624 \pm 0.300$ & 21.80 \\
HPIP & 12 & 11 & $0.602 \pm 0.276$ & 24.56 \\
GPIP & 12 & 12 & $0.529 \pm 0.240^{\mathrm{a}}$ & 33.71 \\
DWPIP & 12 & 12 & $0.601 \pm 0.298$ & 24.69 \\
SPIP & 12 & 11 & $0.576 \pm 0.270$ & 27.57 \\
UPIP & 12 & 11 & $0.800 \pm 0.370$ & 0.00 \\
GPIP+CTX & 12 & 12 & $0.262 \pm 0.104^{\mathrm{b}}$ & 67.11 \\
PSP+CTX & 12 & 12 & $0.294 \pm 0.069^{\mathrm{b}}$ & 63.11 \\
\hline
\end{tabular}

${ }^{\mathrm{a}} \mathrm{P}<0.05$ and ${ }^{\mathrm{b}} \mathrm{P}<0.01$ vs. saline group. $\mathrm{H} 22$-bearing mice were orally administered saline, CTX $25 \mathrm{mg} / \mathrm{kg}$ (intraperitoneal administration, positive control), PSP $200 \mathrm{mg} / \mathrm{kg}$ (positive control) and all types of PIP $200 \mathrm{mg} / \mathrm{kg}$ once daily, for 14 days. Each value is presented as mean \pm standard deviation, $\mathrm{n}=12$. PIP, Phellinus igniarius polysaccharides; CTX, cyclophosphamide; PSP, Trametes versicolor polysaccharopeptide; DMPIP, Dongbei mulberry PIP; HPIP, Hunan mulberry PIP; GPIP, Gansu mulberry PIP; DWPIP, Dongbei white birch PIP; SPIP, Shandong mulberry PIP; UPIP, unknown origin PIP.

$\mathrm{KGaA}$ ) for 3 days and then used in the in vitro experiment. The cultures were maintained at $37^{\circ} \mathrm{C}$ under a humidified $5 \%$ $\mathrm{CO}_{2}$ atmosphere. $\mathrm{H} 22$ cells were maintained as ascites in the KM mice by weekly passage.

In vitro cytotoxic activity. Direct cytotoxicity of GPIP against HepG2 cells was evaluated via MTT assay. Cancer cells were inoculated to 96 well plates at a concentration of 8,000 cells/well and cultured in RPMI-1640 containing $10 \%$ serum for $24 \mathrm{~h}$. Following the addition of 25, 50, 100, 250 and $500 \mu \mathrm{g} / \mathrm{ml}$ GPIP, the cancer cells were further incubated for 24,48 and $72 \mathrm{~h}$ respectively. Then $20 \mu \mathrm{l}$ MTT $(5 \mathrm{mg} / \mathrm{ml})$ was added into each well and incubated for a further $4 \mathrm{~h}$. The supernatant was removed carefully and $150 \mu$ l dimethyl sulfoxide was added to each well. The absorbance at $490 \mathrm{~nm}$ was measured with an ELISA reader. Untreated cells were used as a control with $100 \%$ viability and cells without addition of MTT were used as blank to calibrate the spectrophotometer to zero absorbance. The inhibition ratio (IR) was calculated according to the following formula: IR $(\%)=(1$-absorbance of experimental group/absorbance of blank control group) x 100 .

Cytokine measurements. The concentration of serum IL-2, IL-12 and IFN- $\gamma$ were measured in the normal (non-tumorous, received saline), control, CTX, PSP, GPIP, GPIP + CTX and PSP + CTX groups by a sandwich ELISA method using mouse cytokine ELISA kits (Jinan Rebecca Trading Co.) according to
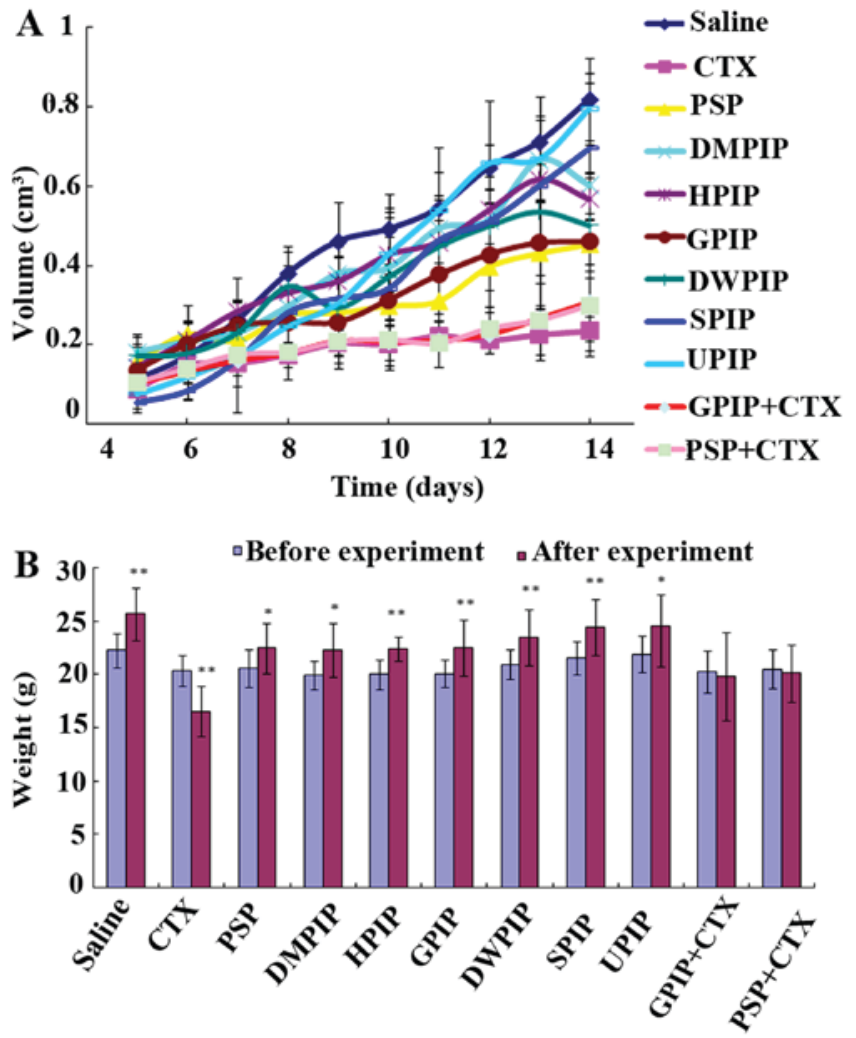

Figure 2. In vivo antitumor effect of PIP. (A) Tumor volume changes in different groups; (B) body weight changes of the mice. ${ }^{*} \mathrm{P}<0.05,{ }^{* *} \mathrm{P}<0.01$ vs. weight before the experiment. PIP, Phellinus igniarius polysaccharides; CTX, cyclophosphamide; PSP, Trametes versicolor polysaccharopeptide; DMPIP, Dongbei mulberry PIP; HPIP, Hunan mulberry PIP; GPIP, Gansu mulberry PIP; DWPIP, Dongbei white birch PIP; SPIP, Shandong mulberry PIP; UPIP, unknown origin PIP.

the manufacturer's protocol. Assays were performed according to the manufacturer's protocol.

Statistical analysis. Treated and control groups were compared using Student's t-test SPSS version 21 (IBM Corp., Armonk, NY, USA) was used for statistical analysis. Results are expressed as means \pm standard deviation. $\mathrm{P}<0.05$ was considered to indicate a statistically significant difference.

\section{Results and Discussion}

Tumor-inhibitory effect of PIP against H22. The in vivo antitumor effect of six different types of PIP was assessed via oral administration using KM mice bearing $\mathrm{H} 22$ tumors as model animals. The tumor weight and inhibition rate are presented in Table I. Among the PIP groups, only the average tumor weight in the GPIP group decreased significantly vs. the saline group $(\mathrm{P}<0.05)$ and the inhibition rate was $33.71 \%$. Furthermore, the tumor weight in the GPIP group had no significant difference compared with PSP positive control group. The tumor volumes in all groups were measured during the experiment. Fig. 2A demonstrates the changes in tumor volumes. It was also identified that the tumor volumes in the GPIP group were smaller than those of other PIP groups, together with the tumor weight changes all indicating that the antitumor effect of GPIP was of the 
Table II. Immune organ indexes in H22-bearing mice $(n=12)$.

\begin{tabular}{lcc}
\hline Groups & $\begin{array}{c}\text { Thymus indexes } \\
\mathrm{mg} / 10 \mathrm{~g}\end{array}$ & $\begin{array}{c}\text { Spleen indexes } \\
\mathrm{mg} / 10 \mathrm{~g}\end{array}$ \\
\hline Control & $29.19 \pm 6.22$ & $44.42 \pm 5.63$ \\
CTX & $13.69 \pm 5.52^{\mathrm{b}}$ & $41.97 \pm 9.40$ \\
PSP & $31.28 \pm 4.68^{\mathrm{d}}$ & $54.71 \pm 4.34^{\mathrm{a}, \mathrm{c}}$ \\
DMPIP & $19.84 \pm 5.33^{\mathrm{a}}$ & $54.66 \pm 9.53$ \\
HPIP & $23.14 \pm 2.12^{\mathrm{d}}$ & $52.52 \pm 7.43$ \\
GPIP & $30.97 \pm 5.45^{\mathrm{d}}$ & $63.40 \pm 8.15^{\mathrm{b}, \mathrm{d}}$ \\
DWPIP & $25.68 \pm 5.69^{\mathrm{d}}$ & $43.12 \pm 12.89$ \\
SPIP & $28.84 \pm 5.90^{\mathrm{d}}$ & $51.26 \pm 8.77$ \\
UPIP & $26.59 \pm 5.24^{\mathrm{d}}$ & $48.03 \pm 9.72$ \\
GPIP+CTX & $17.63 \pm 5.01^{\mathrm{b}}$ & $61.99 \pm 9.07^{\mathrm{b}, \mathrm{d}}$ \\
PSP+CTX & $20.28 \pm 6.79^{\mathrm{a}}$ & $56.30 \pm 9.00^{\mathrm{a}, \mathrm{c}}$
\end{tabular}

${ }^{a} \mathrm{P}<0.05$ and ${ }^{b} \mathrm{P}<0.01$ vs. saline group, ${ }^{\mathrm{C}} \mathrm{P}<0.05$ and ${ }^{\mathrm{d}} \mathrm{P}<0.01$ vs. CTX group. CTX, cyclophosphamide; PSP, Trametes versicolor polysaccharopeptide; PIP, Phellinus igniarius polysaccharides; DMPIP, Dongbei mulberry PIP; HPIP, Hunan mulberry PIP; GPIP, Gansu mulberry PIP; DWPIP, Dongbei white birch PIP; SPIP, Shandong mulberry PIP; UPIP, unknown origin PIP.

most promising among the six different PIP. These results revealed that the antitumor effect was associated with the origin and species. Therefore it was important to study the association between the bioactivity and the origin for better development of PI. Through comparison with other articles, it was identified that PI from different origins had different antitumor activities and the mechanisms were not exactly the same $(9,21)$, which also supported the importance of the present study. Furthermore, in the study from Li et al (9), proteoglycans with different purity or structures exhibited different antitumor activities. Therefore, it was necessary to initially clarify the association between activity and origin, screen the PIP with the best antitumor effect and then further study the structure, because this may save a lot of material resources, manpower, financial resources and improve the efficiency of scientific research.

Fig. 2B demonstrates the body weight changes following the 2 -week experimental period. The results indicated that, with the exception of the CTX group, the body weight in all PIP groups markedly increased following treatment. The analysis of body weight variations may be used to define the systemic toxicity (22). The primary results suggested that PIP was almost non-toxic. CTX, as the chemotherapy drug, served an important role in inhibiting tumor growth, yet its side effects, such as arrest of bone marrow and liver damage, limited its applications. However, the body weight in the two combination groups did not change significantly and thus the results also indicated that GPIP may decrease the toxicity caused by CTX. The safety of PI was the major advantage over conventional chemotherapeutics. The importance of multidisciplinary treatment of cancer in improving patient quality of life has been discussed and the maintenance of quality of life greatly affects decision-making in the therapeutic strategies (23).

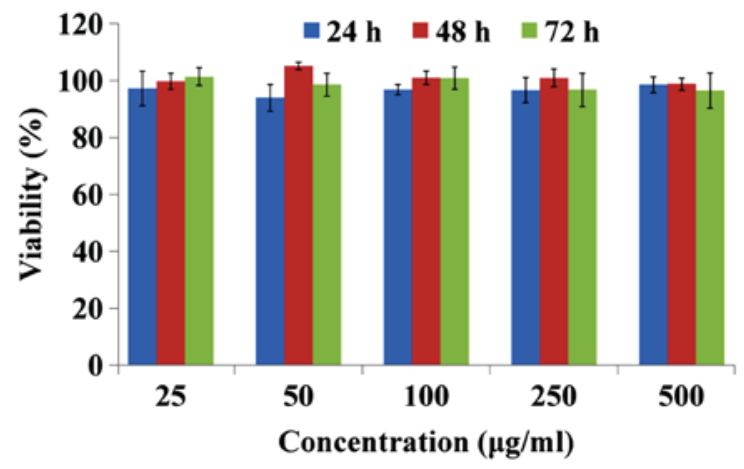

Figure 3. Cytoxicity of GPIP on HepG2 cells. Cells were treated with various concentrations of GPIP $(25,50,100,250$ and $500 \mu \mathrm{g} / \mathrm{ml})$ for 24,48 and $72 \mathrm{~h}$, $(\mathrm{n}=3)$. GPIP, Gansu Phellinus igniarius polysaccharides.

Effect of PIP on immunity. The weight of the thymus and spleen reflects the immune functional strength. In the present study, the relative spleen and thymus index are presented in Table II. GPIP significantly increased the spleen index $(\mathrm{P}<0.01)$ of $\mathrm{H} 22$-bearing mice compared with the saline and CTX group, whereas CTX significantly decreased thymus index $(\mathrm{P}<0.01)$ compared with the saline group. The spleen index in other PIP groups didn't demonstrate any significant increase $(\mathrm{P}>0.05)$ compared with the saline group, which was in accordance with aforementioned antitumor activity. CTX inhibited the growth of tumor, but damaged the immunity of the mice. However, the spleen index in GPIP and CTX combination groups significantly increased compared with CTX group $(\mathrm{P}<0.05)$. The relative spleen and thymus weight were an important index for non-specific immunity. Therefore, these results indicated that GPIP was a potent immunomodulating and immunoenhancing agent, which may enhance the immune function of tumor bearing mice and reduce the immune suppression caused by CTX.

Antitumor mechanism of GPIP. PI has antitumor effects, but the underlying mechanisms responsible for this phenomenon remained controversial. Li et al (11) demonstrated that Phellinus linteus may inhibit the proliferation of HepG2 cells through inducing S-phase arrest. However, different origins and structure of polysaccharides may result in different antitumor mechanism. To further investigate the antitumor mechanism of GPIP, an MTT assay was performed to demonstrate if the antitumor activity of GPIP was induced by direct cytotoxicity. The results are presented in Fig. 3 and indicate that GPIP exhibited no significant cytotoxicity on HepG2 cells at any concentration or time point. Therefore, the antitumor mechanism of GPIP may not be due to inhibition of tumor proliferation directly.

In view of the aforementioned influence on the immune organs, GPIP may enhance the immune function. Therefore, it was speculated that the antitumor mechanisms of GPIP may be associated with the enhancement of immune function. Macrophages and lymphocytes belong to two major populations of cells in the host defense system, which act against invading pathogens. IFN- $\gamma$ and IL- 2 are secreted by type 1 helper T-cells (Th1 cells) and mediate cellular immunity. IFN- $\gamma$ performs a tumoricidal activity and induces other cells of the innate immune system, including macrophages and dendritic cells 


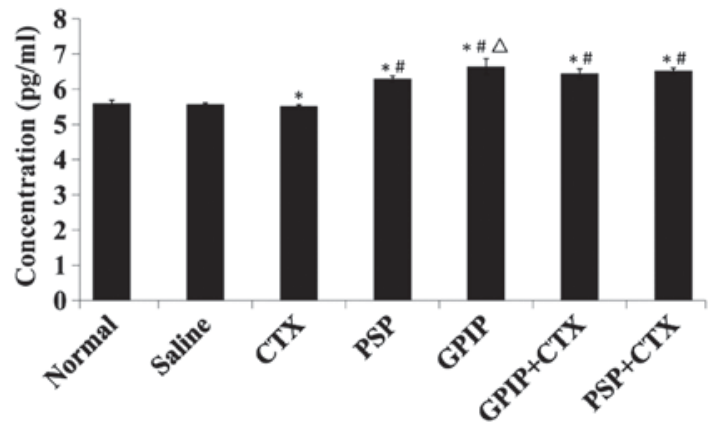

Figure 4. Serum IL-2 in H22-bearing mice. H22-bearing mice were administered saline (negative control group), saline (normal group, non-tumorous), CTX, PSP, GPIP, GPIP + CTX and PSP + CTX once daily, for 14 days. Sera were collected from the tumor-bearing mice on day 15. IL-2 concentration was determined using ELISA method $(n=3)$. * $\mathrm{P}<0.01$ vs. saline group, ${ }^{\#} \mathrm{P}<0.01$ vs. CTX group, and ${ }^{\Delta} \mathrm{P}<0.05$ vs. PSP group. IL-2, interleukin-2; CTX, cyclophosphamide; PSP, Trametes versicolor polysaccharopeptide; GPIP, Gansu mulberry Phellinus igniarius polysaccharides; ELISA, enzyme-linked immunosorbent assay.

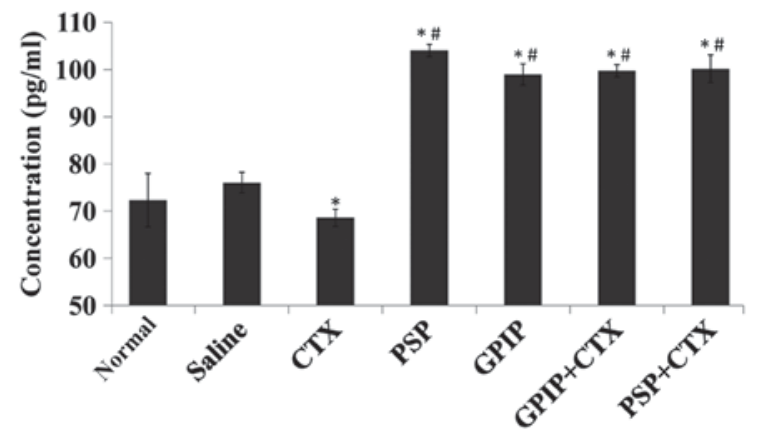

Figure 5. Serum IL-12 in H22-bearing mice. H22-bearing mice were administered saline (negative control group), saline (normal group, non-tumorous) CTX, PSP, GPIP, GPIP + CTX and PSP + CTX once daily, for 14 days. Sera were collected from the tumor bearing mice on day 15 . IL-12 concentration was determined using ELISA method $(n=3)$. " $\mathrm{P}<0.01$ vs. saline group ${ }^{\#} \mathrm{P}<0.01$ vs. CTX group. IL-12, interleukin-12; CTX, cyclophosphamide; PSP, Trametes versicolor polysaccharopeptide; GPIP, Gansu mulberry Phellinus igniarius polysaccharides; ELISA, enzyme-linked immunosorbent assay.

(DCs), to produce IL-12, which further activates cells mediating the innate response (24). A number of studies have demonstrated that IFN- $\gamma$, IL-12, and IL-2 have a function in the immunomodulation of polysaccharides $(3,4)$ and the determination of cytokine concentration was a simple approach to characterize changes in immune function. Therefore, the serum level of IL-2, IL-12 and IFN- $\gamma$ was measured in tumor-bearing mice to further demonstrate the antitumor mechanisms of GPIP. The results for the concentration of IL-2, IL-12 and IFN- $\gamma$ are presented in Figs. 4-6, respectively. With GPIP stimulation, the serum levels of the three cytokines significantly increased compared with the saline and CTX groups $(\mathrm{P}<0.01)$. Furthermore, the IL-2 in GPIP group exhibited a significant increase compared with the PSP group $(\mathrm{P}<0.05)$. IL-2 may stimulate the reactivity of numerous types of killer cells, such as natural killer (NK) cells and cytolytic T lymphocytes $(21,25)$, thus the high level of IL-2 enhanced the cytolytic activity of NK cells and cytolytic T lymphocytes. The trend for the secretion of IFN- $\gamma$ was the same as that observed for the secretion of IL-12. IL-12 is one

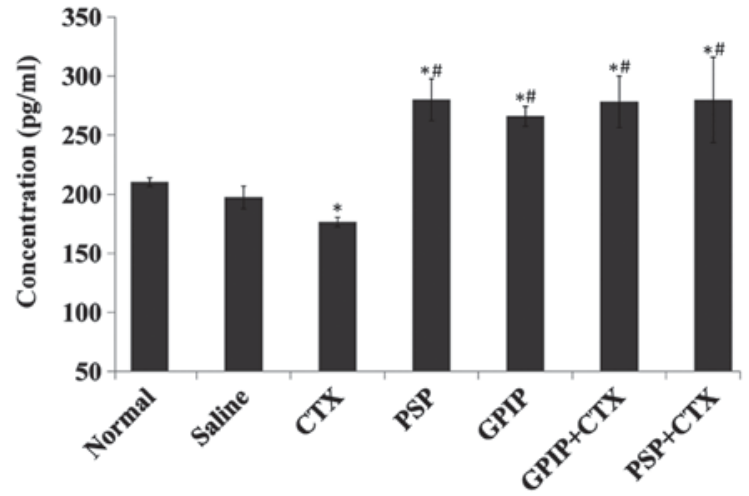

Figure 6. Serum IFN- $\gamma$ in H22-bearing mice. H22-bearing mice were administered saline (negative control group), saline (normal group, non-tumorous), CTX, PSP, GPIP, GPIP + CTX and PSP + CTX once daily, for 14 days. Sera were collected from the tumor bearing mice on day 15 . IFN- $\gamma$ concentration was determined using ELISA method $(\mathrm{n}=3)$. ${ }^{*} \mathrm{P}<0.01$ vs. saline group, ${ }^{\#} \mathrm{P}<0.01$ vs. CTX group. IFN- $\gamma$, interferon- $\gamma$; CTX, cyclophosphamide; PSP, Trametes versicolor polysaccharopeptide; GPIP, Gansu mulberry Phellinus igniarius polysaccharides; ELISA, enzyme-linked immunosorbent assay.

of the crucial cytokines for transition of the immune response from innate to adaptive as the antigen-presenting cells, such as monocytes and DCs produce a substantial amount of IL-12 that primes the Th1 response (26). This in turn induces the production of IFN- $\gamma$ and IL-12 by the Th1 cells to further stimulate downstream cell-mediated immunity (27), which may explain the consistency of IL-12 and IFN- $\gamma$ in the present study. Furthermore, the three cytokines in GPIP and CTX combination groups also exhibited a significant increase $(\mathrm{P}<0.01)$ compared with CTX, which decreased the level of all these cytokines significantly $(\mathrm{P}<0.01)$. The results indicated that GPIP was not only an immune enhancing agent but also may decrease the immunosuppression caused by CTX. According to these results, GPIP was an effective immunopotentiator and the antitumor mechanism may be associated with the enhancement of immune function, which was regulated by GPIP. In conclusion, GPIP was a promising plant polysaccharide as an immunoregulator that demonstrated great potential in the treatment of cancer.

Hepatocellular carcinoma is one of the most prevalent malignant tumors worldwide, and has an extremely poor prognosis. The results of the present study suggest that PIP may have potential therapeutic applications in a clinical setting. Such immune regulatory effects of PIP should be further investigated to identify how the components of the PIP interact with immune cells.

\section{Acknowledgements}

The present study was supported by the Development of Science and Technology Plan Project of Shandong Province (grant no. 2012GSF1191), Jinan Science and Technology Project (grant no. 201101025).

\section{References}

1. Sliva D, Jedinak A, Kawasaki J, Harvey K and Slivova V: Phellinus linteus suppresses growth, angiogenesis and invasive behaviour of breast cancer cells through the inhibition of AKT signalling. Br J Cancer 98: 1348-1356, 2008. 
2. Chen Y, Wan Y, Wang Y, Zhang H and Jiao Z: Anticancer efficacy enhancement and attenuation of side effects of doxorubicin with titanium dioxide nanoparticles. Int J Nanomedicine 6: 2321-2326, 2011

3. Sun Y, Sun T, Wang F, Zhang J, Li C, Chen X, Li Q and Sun S: A polysaccharide from the fungi of Huaier exhibits anti-tumor potential and immunomodulatory effects. Carbohydr Polym 92: $577-582,2013$

4. Yi Y, Zhang MW, Liao ST, Zhang RF, Deng YY, Wei ZC, Tang XJ and Zhang Y: Structural features and immunomodulatory activities of polysaccharides of longan pulp. Carbohydr Polym 87: 636-643, 2012

5. Fabricant DS and Farnsworth NR: The value of plants used in traditional medicine for drug discovery. Environ Health Perspect 109 Suppl 1: S69-S75, 2001.

6. Gao C, Zhong L, Jiang L, Geng C, Yao X and Cao J: Phellinus linteus mushroom protects against tacrine-induced mitochondrial impairment and oxidative stress in HepG2 cells. Phytomedicine 20: 705-709, 2013.

7. Zhao R, Gao X, Cai Y, Shao X, Jia G, Huang Y, Qin X, Wang J and Zheng X: Antitumor activity of Portulaca oleracea L. polysaccharides against cervical carcinoma in vitro and in vivo. Carbohydr Polym 96: 376-383, 2013.

8. Zou X, Sun M and Guo X: Quantitative response of cell growth and polysaccharide biosynthesis by the medicinal mushroom Phellinus linteus to $\mathrm{NaCl}$ in the medium. World J Microb Biot 22 1129-1133, 2006.

9. Li X, Jiao LL, Zhang X, Tian WM, Chen S and Zhang LP. Anti-tumor and immunomodulating activities of proteoglycans from mycelium of Phellinus nigricans and culture medium. Int Immunopharmacol 8: 909-915, 2008.

10. He P, Geng L, Wang J, Wang Z, Mao D and Xu C: Purification, characterization and bioactivity of an extracellular polysaccharide produced from Phellinus igniarius. Ann Microbiol 62 1697-1707, 2012.

11. Li YG, Ji DF, Zhong S, Liu PG, Lv ZQ, Zhu JX, Chen JE and Chen HP: Polysaccharide from Phellinus linteus induces S-phase arrest in HepG2 cells by decreasing calreticulin expression and activating the P27kip1-cyclin A/D1/E-CDK2 pathway. J Ethnopharmacol 150: 187-195, 2013.

12. Zhong S, Ji DF, Li YG, Lin TB, Lv ZQ and Chen HP: Activation of P27kip1-cyclin D1/E-CDK2 pathway by polysaccharide from Phellinus linteus leads to S-phase arrest in HT-29 cells. Chem Biol Interact 206: 222-229, 2013.

13. Zong A, Cao H and Wang F: Anticancer polysaccharides from natural resources: A review of recent research. Carbohydr Polym 90: 1395-1410, 2012.

14. Wu SJ, Liaw CC, Pan SZ, Yang HC and Ng LT: Phellinus linteus polysaccharides and their immunomodulatory properties in human monocytic cells. J Funct Foods 5: 679-683, 2013.
15. Blattman JN and Greenberg PD: Cancer immunotherapy: A treatment for the masses. Science 305: 200-205, 2004.

16. Gajewski TF, Woo SR, Zha Y, Spaapen R, Zheng Y, Corrales L and Spranger S: Cancer immunotherapy strategies based on overcoming barriers within the tumor microenvironment. Curr Opin Immunol 25: 268-276, 2013.

17. Landskron G, De la Fuente M, Thuwajit P, Thuwajit $C$ and Hermoso MA: Chronic inflammation and cytokines in the tumor microenvironment. J Immunol Res 2014: 149185, 2014.

18. Li Xiong S, Li A, Huang N, Lu F and Hou D: Antioxidant and immunoregulatory activity of different polysaccharide fractions from tuber of Ophiopogon japonicus. Carbohydr Polym 86: 1273-1280, 2011

19. Jeff IB, Yuan X, Sun L, Kassim RM, Foday AD and Zhou Y: Purification and in vitro anti-proliferative effect of novel neutral polysaccharides from Lentinus edodes. Int J Biol Macromol 52: 99-106, 2013

20. Mou ZZ, Wang MF, Gao WW, Zhang N and Yu SW: Extraction of Phellinus igniarius polysaccharides and composition analysis of monosaccharide. Chin J Experimental Traditional Medical Formulae 20: 13-15, 2014 (In Chinese).

21. Chen L, Pan J, Li X, Zhou Y, Meng Q and Wang Q: Endo-polysaccharide of Phellinus igniarius exhibited anti-tumor effect through enhancement of cell mediated immunity. Int Immunopharmacol 11: 255-259, 2011.

22. Huang HY, Chieh SY, Tso TK, Chien TY, Lin HT and Tsai YC: Orally administered mycelial culture of Phellinus linteus exhibits antitumor effects in hepatoma cell-bearing mice. J Ethnopharmacol 133: 460-466, 2011

23. De Silva DD, Rapior S, Fons F, Bahkali AH and Hyde KD Medicinal mushrooms in supportive cancer therapies: An approach to anti-cancer effects and putative mechanisms of action. Fungal Diversity 55: 1-35, 2012.

24. Wong KH,Lai CK and Cheung PC: Immunomodulatory activities of mushroom sclerotial polysaccharides. Food Hydrocolloids 25: $150-158,2011$.

25. Xie G, Schepetkin IA and Quinn MT: Immunomodulatory activity of acidic polysaccharides isolated from Tanacetum vulgare L. Int Immunopharmacol 7: 1639-1650, 2007.

26. Cella M, Scheidegger D, Palmer-Lehmann K, Lane P, Lanzavecchia A and Alber G: Ligation of CD40 on dendritic cells triggers production of high levels of interleukin-12 and enhances T cell stimulatory capacity: T-T help via APC activation. J Exp Med 184: 747-752, 1996.

27. Qin $\mathrm{Z}$ and Blankenstein T: $\mathrm{CD}^{+}{ }^{+} \mathrm{T}$ cell-mediated tumor rejection involves inhibition of angiogenesis that is dependent on IFN gamma receptor expression by nonhematopoietic cells. Immunity 12: 677-686, 2000 